\title{
Coexistence of Cloud Technology and IT Infrastructure in Higher Education
}

\author{
Laurynas Riliskis, Evgeny Osipov \\ Department of Computer Science, Electrical and Space Engineering \\ Luleå University of Technology \\ 97187 Luleå, Sweden \\ laurynas.riliskis@ltu.se
}

\begin{abstract}
Early 2012 Luleå University of Technology started a project on adopting cloud technology for implementation in the university's IT-infrastructure. This work-in-progress article describes the results of its pre-study phase aiming at understanding the feasibility of integrating and/or migrating main IT-infrastructure components into an IaaS system and opening ways for making university's resources more accessible to a wider public. Numbers of logistical, technical and education related challenges make such transition far from being trivial. The article focuses on the educational aspect of the pre-study. Specifically, work flows in education process of several courses in different disciplines in natural and engineering sciences were analyzed from the student and teacher perspectives. In the article a schematic of a sustainable IT infrastructure adjusted to the needs of higher education will be drafted. Further, technical readiness and challenges of using cloud technology for university scale IT-infrastructure are discussed.
\end{abstract}

\section{INTRODUCTION}

Most of the current IT-infrastructures at Universities were designed and deployed at times of mostly campus-based education, following the "One-Solution- Fits-All" approach. Nowadays, with the growing number of courses given on distance and unique requirements on software content in almost every course in all disciplines the existing infrastructure became extremely stiff and inefficient. In many cases it fails providing flexible services with an acceptable quality both to teachers and students. Another problem appears with the all growing mobility of students: The uncertainty in number of students to be handled in each semester (even in the scope of a particular course) makes it difficult for the IT departments to estimate the need and to correspondingly dimension the IT-system. Normally, university's IT administrations over-dimension their infrastructures in order to stay competitive and provide a satisfactory quality of experience. This obviously leads to an increased cost associated with purchasing and maintaining the equipment. Besides the economical component the environmental impact becomes one of the critical factors that universities have to deal with nowadays. Universities as governmental organizations need to take environmental considerations into account when implementing European Union policies on ICT sustainability. Cloud technology and specifically the infrastructure as a service (IaaS) modality is an excellent alternative to an outdated and inefficient local infrastructures.

For Luleå Technical University (LTU ${ }^{1}$ ) with seventeen thousand students and four thousand stuff members the de-

\footnotetext{
${ }^{1}$ http://www.ltu.se
}

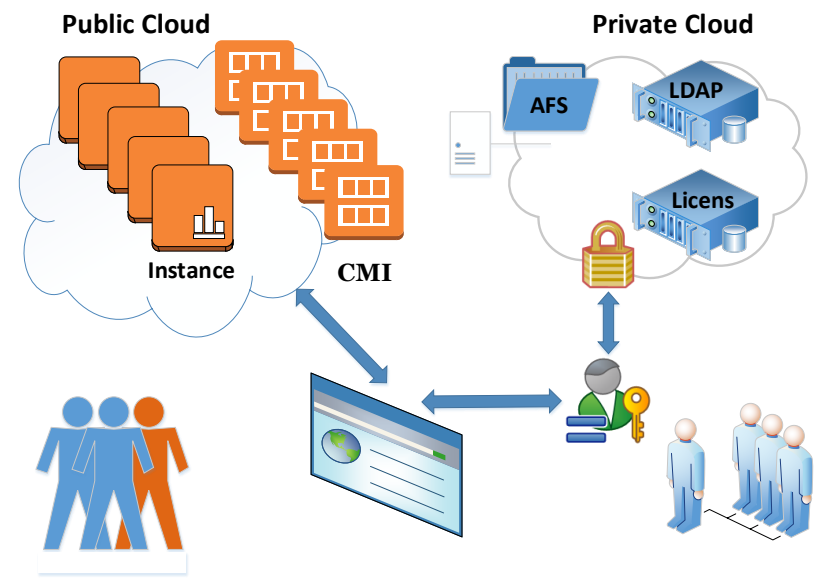

Fig. 1. Envisioned future infrastructure for higher education.

mand on the functional IT infrastructure is high. LTU is located in five geographically distributed campus areas, with each campus having both a general purpose and a specialized computer set-ups. Besides the standard set of applications as in a general purpose $\mathrm{PC}$, the PC's in specialized labs have extra hardware and/or software depending on the course requirements. Currently more than four hundred different applications has to coexist in different configurations in order to satisfy students' needs and teachers' requirements on educational software. To ensure the compatibility of applications the requirements are collected by IT administration one semester before the course start, the compatibility is tested and custom installation images are created. Before the start of a new semester the customized images are deployed. To preserve the usability of the PC labs for students and teachers the deployment usually takes place during weekends and nights. Overall this process is expensive and not flexibility for teachers and students.

While the idea of using cloud technology in education is not new [1], the related existing work is often limited to courses related to cloud computing [2], [3] or e-Education [4] including the well-known success story of EdX [5]. Compared to other works on education in the cloud we address the needs for flexible IT infrastructure from the teachers, students and IT administrators perspective.

The on-going project on adopting the cloud technology for implementing the IT-infrastructure at LTU has the following 
objectives:

a) Students should be able access computing resources from anywhere, any time;

b) Students should be able to customize the working environment using administrator rights;

c) Teachers should be able to influence the setup of the computing resources both before and after the course start;

d) The solution should be scalable and energy efficient;

e) The solution should minimize the human resources needed to deploy, set up and maintain the IT platform for the course.

This work-in-progress article describes the results of the pre-study phase of the project aiming at understanding the feasibility of integrating and/or migrating the main IT infrastructure components to the cloud as well as drafting the initial design of the architecture.

\section{Vision OF A SUSTAINABLE ITS}

In order to increase the flexibility of the IT infrastructure both for teachers and students we envision a scenario where each course with standard hardware requirements owns a unique software solution in a form of a dedicated virtual machine image. Further we refer this dedicated virtual machine image as course virtual image or $C V I$. This image can be started as an instance in the cloud environment or downloaded for usage on a local computer with an available virtualization software, for example such as Virtual Box [6] or VMware [7].

Figure 1 depicts the envisioned "one-course-one-CVI" scenario. The infrastructure is a hybrid cloud solution where the private cloud is maintained on the local IT infrastructure and the public cloud is outsourced to an infrastructure as a service (IaaS) provider. The private cloud contains services for student authentication (LDAP [8]), application licensing service (License), and distributed files system (AFS [9]). On the public cloud the course's specific CVI's are stored and run by students or staff. Students access the pre-deployed CVI from the course web page, by choosing either to launch an instance or download the image for local usage. The students' global home directory is added as a network-mapped drive to the instance. Therefore, students can store their files across all CVIs. After the CVI started, students gain administrator rights on the instance and are able to further configure it and install additional software. When students log out, the instance is stopped and resources are not wasted unnecessary.

\section{IMPROVED PROCESS OF MANAGING ITS RESOURCES}

The current process of deploying software needed for courses is challenging. The IT service collects teachers request for the course specific software one semester in advance. The software is installed and the interoperability is tested in a dedicated testbed. The ITS personnel needs to have a deep expertise in all the deployed software to test the functionality. whenever changes in the software are needed shortly before or during the course it becomes an expensive matter for the IT administration. We advocate that this situation could be substantially improved. The envisioned future process is illustrated in Figure 2, a teacher makes a request to the local IT administrator specifying course computational need including

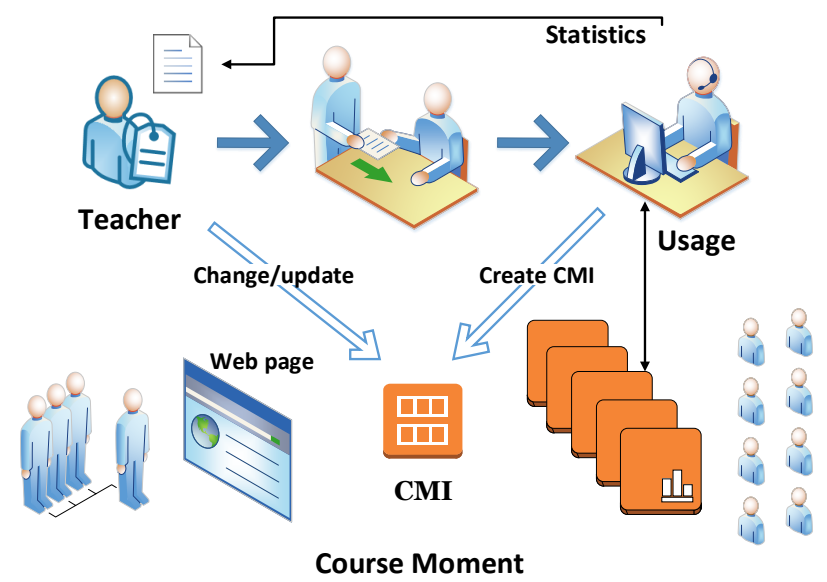

Fig. 2. A process for the creation of course CMI in the Clouds Infrastructure.

for the course needed software. The CVI is created by the IT administration and is deployed in the public cloud. Since none of the other courses would be affected, if needed, the teacher could further customize the CVI by herself. The access to CVI is enabled from the course's web page for the students. This approach not only makes the infrastructure easily maintainable and flexible but also removes the following human resource consuming steps:

- Tests related to software interoperability between different courses;

- Resolution of conflicts between different software;

- Deployment of the software on the physical machines;

- Management of student access rights on the lab computers.

From the teacher perspective the "one-course-one-CVI" approach gives valuable insights on the students' work load distribution during the course. This is achieved by monitoring the activity of instances within the particular course. As a matter of fact, the billing of the resource usage is based on the per-hour usage of the instances. Thus anonymous (no student names are connected to a particular instance) statistics are available on monthly bases from the public cloud provider which can be used to analyze the work load.

\section{EXPERIENCE AND FEASIBILITY}

As part of the project the IT needs of several courses were powered by Amazon Web Services cloud infrastructure [10]. Amazon AWS was selected as cloud service provider because it offered the desired programing flexibility and provided educational grants.

The feasibility testing was performed outside the current IT infrastructure. Three courses within computer science education were selected for the test group. Prior to the start of the courses a front-end was developed for generating login credentials associated with usage of Amazon AWS, and for managing the AWS resource usage. The CVI as created containing the necessary software for the course. 
So far the teachers' and the students' experiences with the in-cloud IT infrastructure were positive. From the teachers and students perspective the biggest value was a ability to personalize the working environment as well as an ability to access courses' software at anytime from any location in a straightforward way. The IT administration of the course for the deployment of needed software was minimal. Several onfly updates of the software were performed during the course which also required minimal effort from the IT administration. On the criticism side, the students using remote desktop often experienced lag. While the quality of experience could be to some extend improved by selecting a more computationally powerful virtual machine, the network delays were in many cases dominant.

The teachers highlighted advantages of cloud-based infrastructure in terms of a flexible software set up and prior to all an ability to deploy new version during the course's run. The CVI was updated and new version deployed in a matter of ten minutes. Additionally, the transition was transparent to the students without disturbing the learning process.

After a week of usage we could notice that students were not properly turning off the instances and they were left running during long time periods. Therefore an application for an individual resource usage monitoring and automating the process of stopping the instances was developed. Even though this costs additional work for the IT administrators, once deployed this add-on did not require additional maintenance. It also became clear that providing valuable statistics of resource usage to the teacher was not a trivial task and required development of customized tools.

\section{Challenges and Future Work}

The benefits of using cloud infrastructure in higher education are evident, the important ones are: extreme flexibility when setting up the course software, the course can be given to any number of students, the course's software is accessible from anywhere either online or locally. Numbers of logistical, technical and education-related challenges make, however, such transition far from being trivial. To the best of our knowledge there is no user and cloud management application for the integrated environment of the hybrid cloud together with LDAP, AFS and Licensing services. Such application, nevertheless, is feasible to develop on-cite. One of the next steps in the project is an investigation of economical cost for such development.
The most challenging aspect to overcome when adopting the cloud-based IT infrastructure for education is legal issues. Firstly, licensing of software used in the courses is vendor specific. While some software vendors use per-user licensing, other require users to be located on campus which makes the situation problematic. Secondly, the legal aspects related to privacy and national education laws and regulations are not obvious to resolve when matter comes to deploying the educational infrastructure to as hybrid cloud. While there are many studies about privacy in the cloud (see also references therein) our proposed solution overcomes many of them because student identification, authentication as well as their private files are stored in local infrastructure. We, however, foresee a substantial work load on the legal department in order to determine the appropriate setup.

So far we are optimistic that the benefits provided by our "one-course-one-CVI" vision outweighs the legal and logistical challenges. The next step in our work will be to make economical and legal analysis of the project as well as to deploy a pilot setup and to evaluate students' experience on a bigger scale.

\section{REFERENCES}

[1] N. Sultan, "Cloud computing for education: A new dawn?" International Journal of Information Management, vol. 30, no. 2, pp. 109-116, 2010. [Online]. Available: http://www.sciencedirect.com/ science/article/pii/S0268401209001170

[2] S. Yokoyama, N. Yoshioka, and T. Shida, "Edubase cloud: Cloud platform for cloud education," in Software Engineering Education based on Real-World Experiences (EduRex), 2012 First International Workshop on, 2012, pp. 17-20.

[3] [Online]. Available: http://www.usfca.edu/analytics/

[4] P. Soni and P. Gupta, E-Learning Through Cloud Computing: Shaping the Future of Learning for Learner of Tomorrow, B. Xu, Ed. Springer Berlin Heidelberg. [Online]. Available: http://dx.doi.org/10. 1007/978-3-642-34910-2_56

[5] [Online]. Available: https://www.edx.org/

[6] [Online]. Available: https://www.virtualbox.org/

[7] [Online]. Available: http://www.vmware.com/

[8] "Lightweight Directory Access Protocol," June 2006. [Online]. Available: http://tools.ietf.org/html/rfc4510

[9] J. H. Howard, M. L. Kazar, S. G. Menees, D. A. Nichols, M. Satyanarayanan, R. N. Sidebotham, and M. J. West, "Scale and performance in a distributed file system," ACM Trans. Comput. Syst., vol. 6, no. 1, pp. 51-81, Feb. 1988. [Online]. Available: http://doi.acm.org/10.1145/35037.35059

[10] [Online]. Available: https://aws.amazon.com/ 